\title{
Intralobar pulmonary sequestration
}

\author{
W. B. JAMES \\ M.B., F.F.R., D.M.R.D.
}

\author{
R. J. CUTHBERT \\ B.Sc., M.B., D.P.H., F.R.C.P.(Glas.), F.R.C.P.(Edin.)
}

Departments of Radiology and Medicine, Southern General Hospital, Glasgow

\section{Introduction}

Pulmonary sequestration is an uncommon condition. It occurs in both an extralobar and an intralobar form. Extralobar pulmonary sequestration is rare and consists of an accessory ectopic lobe lying outside the normal lung enclosed in its own visceral pleura. The commoner intralobar form lies within the visceral pleura, usually in the lower lobe posteriorly and on the left side. The blood supply arises not from the pulmonary artery but from the descending thoracic or upper abdominal aorta. The sequestrated segment does not have a normal connection with the bronchial system of the lobe with which it is associated but infection commonly occurs, tends to be chronic and recurrent and is usually the presenting feature (Le Roux, 1962). While suspicion may be aroused by the appearances on plain radiography, tomography and bronchography, a definitive diagnosis can be made only by demonstrating the abnormal arterial supply at aortography (Turk \& Lindskog, 1961 ; Sutton \& Samuel, 1963 ; Neilsen, 1964 ; Ranniger \& Valvassori, 1964). In addition arteriography will demonstrate the number of anomalous arteries and their sites of origin from the aorta.

Four cases of intralobar pulmonary sequestration are presented. In two of these cases the diagnosis was not suspected pre-operatively: in the other two the diagnosis was suggested preoperatively and in one aortography and selective arteriography of the abnormal vessel enabled a definitive diagnosis to be made.

\section{Case reports}

\section{Case 1}

A married woman aged 40 presented in 1952 with a chronic cough and sputum which had been worse in the previous 4 years. She gave a long history of chest trouble and on at least six occasions had been admitted to hospital with pneumonia. Chest radiography revealed a large rounded opacity containing a fluid level at the left base posteriorly (Fig. 1). A diagnosis of pulmonary abscess was made. Shortly after admission the patient developed signs of cerebellar dysfunction -ataxia, vertigo, nystagmus and past-pointing. After intensive treatment with penicillin and chloramphenicol the abnormal neurological signs disappeared. The 'lung abscess' underwent marked shrinkage. Bronchography demonstrated that the abscess communicated with the bronchial tree and that there were bronchiectatic changes in the left lower lobe (Fig. 2). A diagnosis of simple lung abscess secondary to bronchiectasis was made and thoracotomy was carried out.

At operation the posterior basal segment of the lung was adherent to the aorta and at first this was thought to be due to inflammatory reaction.

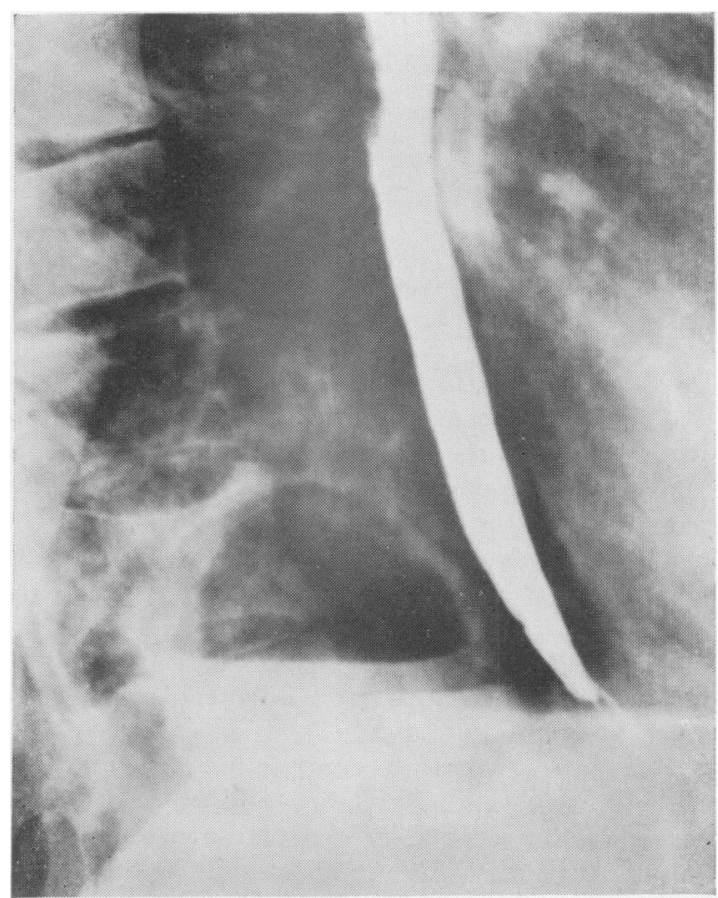

Fig. 1. (Case 1). Left posterior basal opacity with fluid level. Barium in oesophagus. 


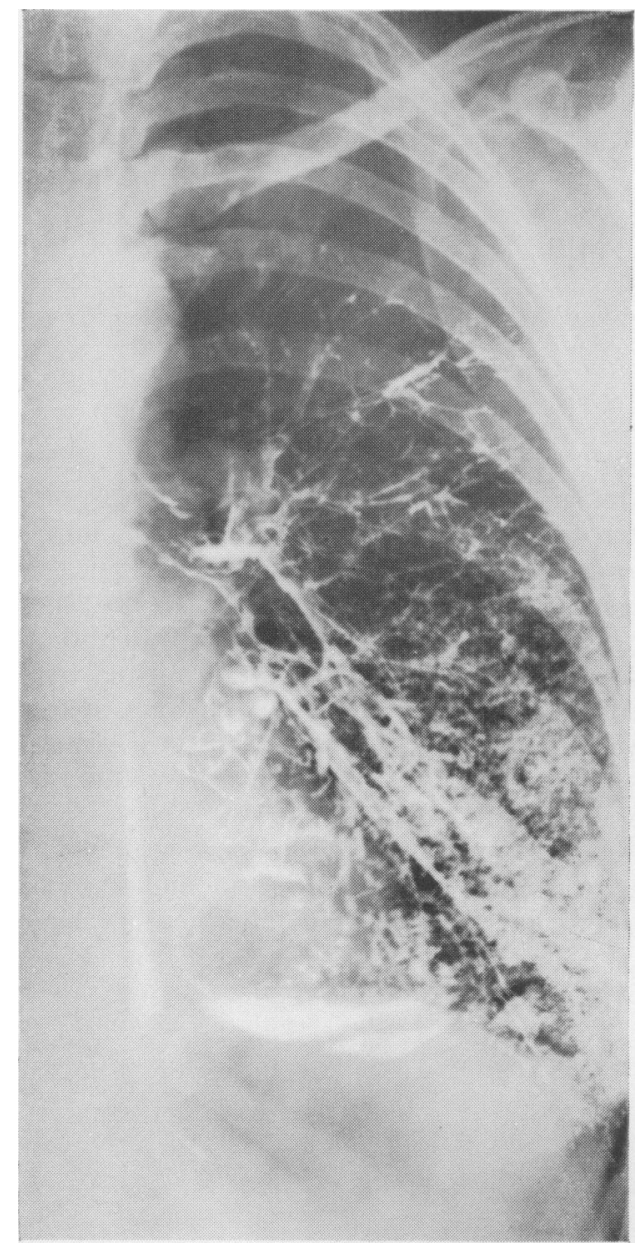

Fig. 2. (Case 1). Bronchography left lower lobe. The cyst cavity communicates with the bronchial tree.

In the course of separating adhesions, massive haemorrhage occurred and was found to come from one of three abnormal vessels arising from the aorta and passing directly to what was now recognized as a sequestrated lung segment. The two intact vessels were ligated and divided and lobectomy of the lower lobe completed. The torn abnormal vessel was then defined. The tear had occurred at the origin of the vessel and was controlled by suture-this reduced the lumen of the aorta by about $50 \%$.

The patient made a good immediate recovery from the operation but the following day there was clinical evidence of coarctation of the aorta -hypertension in the upper limbs $(180 / 80 \mathrm{mmHg}$ compared with $130 / 80 \mathrm{mmHg}$ before operation) and impaired circulation in the lower limbs (absent pulses, coldness, cramp-like pains and diminished sensation). Two days later cardiac failure developed and the patient died on the 6th post operative day.

Comment. This case illustrates a danger if catastrophic haemorrhage occurs when the diagnosis has not been clear before operation. Similar cases have been described by Harris \& Lewis (1940) and Douglass (1948). The abnormal artery supplying the sequestration usually runs along the pulmonary ligament and access to this region is not always easy, particularly in the presence of infection. Inflammatory adhesions may obscure the vessel and accompanying fibrosis may make haemorrhage difficult to control (Pryce, Sellors \& Blair, 1947).

\section{Case 2}

A 37-year-old married woman was referred when a routine pre-employment chest radiograph revealed a circumscribed opacity in the posterior basal segment of the left lower lobe behind the cardiac shadow in the PA film. There was no history of previous chest trouble. Lateral tomography confirmed the presence of a well-defined lobulated mass (Fig. 3). Bronchography showed slight lateral displacement of the lower lobe

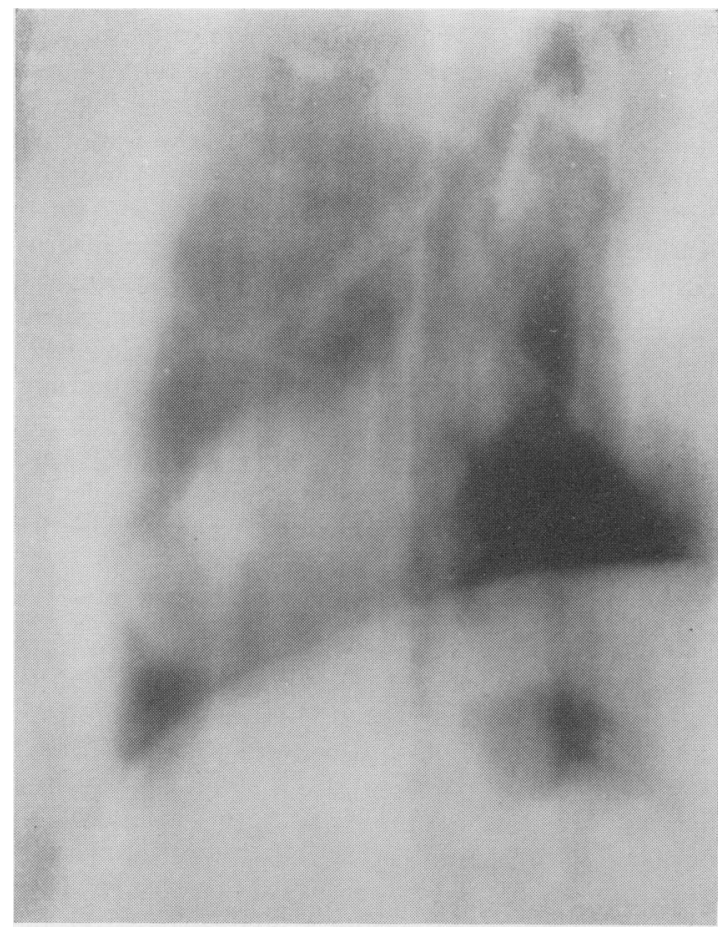

FIG. 3. (Case 2). Lateral tomography confirms the presence of a well defined, lobulated mass at the left base posteriorly. 
bronchi. There was no evidence of a bronchial communication with the lesion. Bronchoscopy showed no abnormality. A diagnosis of probable pulmonary sequestration was made.

Thoracotomy revealed a soft cyst-like swelling in the posterior basal segment of the left lower lobe and dissection confirmed this to be a sequestrated segment. The abnormal segment was excised after ligation and division of its abnormal supplying artery which arose from the dorsal aorta. The patient made an uneventful recovery. Histological examination of the excised specimen confirmed the nature of the lesion as a sequestrated lung segment containing a large thick walled cyst filled with pus.

Comment. This case illustrates two points: (a) Most patients with pulmonary sequestration present with chronic or recurrent chest infection but the absence of such a history, even in an adult patient, does not invalidate the diagnosis. (b) The sequestrated segment rarely communicates with the bronchial tree. As a result, no filling of the lesion is seen on bronchography and the adjacent normal bronchi appear displaced. Such appearances should raise the suspicion of sequestration.

\section{Case 3}

A 62-year-old married woman had for 6 years been troubled with recurrent respiratory infection, worse during the last 12 months. Chest radiography demonstrated a rounded opacity in the posterior basal segment of the right lower lobe (Fig. 4). A

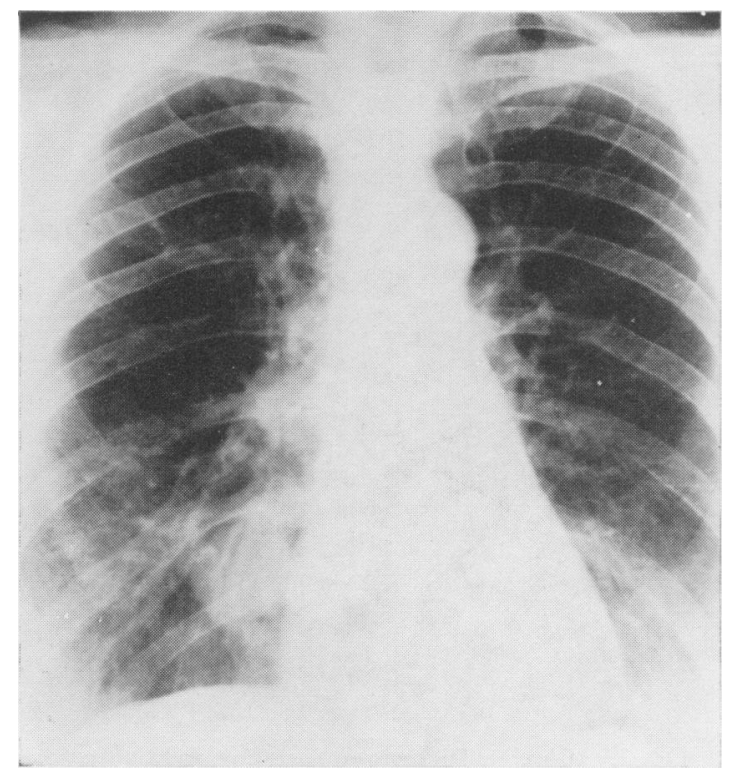

Fig. 4. (Case 3). P.A. radiograph shows a 3-cm spherical opacity at the right base. provisional diagnosis of bronchogenic carcinoma was made. At thoracotomy a rather soft partly cystic mass was found. Dissection of the affected segment revealed its blood supply to be directly from the dorsal aorta by an artery of the calibre of a brachial artery. Right lower lobectomy was carried out. The patient made an uneventful recovery.

The intralobar sequestrated segment consisted of a cyst $3 \mathrm{~cm}$ in diameter, filled with mucoid material and lined by a single layer of primitive low columnar epithelium which in many areas was ciliated. The abnormal artery had an extremely thick muscular wall and pursued a very tortuous course. One large branch showed partial recanalization of old thrombus.

Comment. Left basal intrapulmonary sequestration is at least twice as common as that on the right (Turk \& Lindskog, 1961). Nearly all of the reported cases in the literature presented at a much earlier age than in this patient. For these reasons the diagnosis had not been considered.

\section{Case 4}

A 35-year-old married woman attended with a complaint of blood stained sputum over a period of 1 month. She had had one previous episode of haemoptysis 3 years before. There was no history of any other chest illness and she felt well. Chest radiography showed a small ill-defined triangular opacity seen through the left heart shadow (Fig. 5). In retrospect this shadow although visible on a chest film taken 3 years earlier had not excited comment. Fluoroscopy and tomography (Fig. 6) demonstrated the lesion more clearly and showed it to lie posteriorly. Bronchography showed no significant abnormality. A provisional diagnosis of sequestrated segment was made and percutaneous transfemoral aortography was carried out for confirmation. After a test injection a large abnormal artery was seen to arise from the lower descending thoracic aorta (Fig. 7). The catheter was then changed and a curved selective catheter was guided into the abnormal artery. The selective arteriogram showed more clearly the anatomy of the arterial supply and its venous drainage (Fig. 8). These findings were confirmed at thoracotomy. The sequestrated segment was adherent to the left lower lobe and left lower lobectomy had to be performed. The specimen consisted of a large cyst filled with caramel coloured semi-fluid, necrotic material and surrounded by greyish tissue. Its walls contained a considerable amount of fibrous tissue and the lining was of ciliated bronchial epithelium. An unusual and interesting feature in this case was the presence in the sequestrated tissue of numerous typical caseating tuberculous follicles. The anoma- 


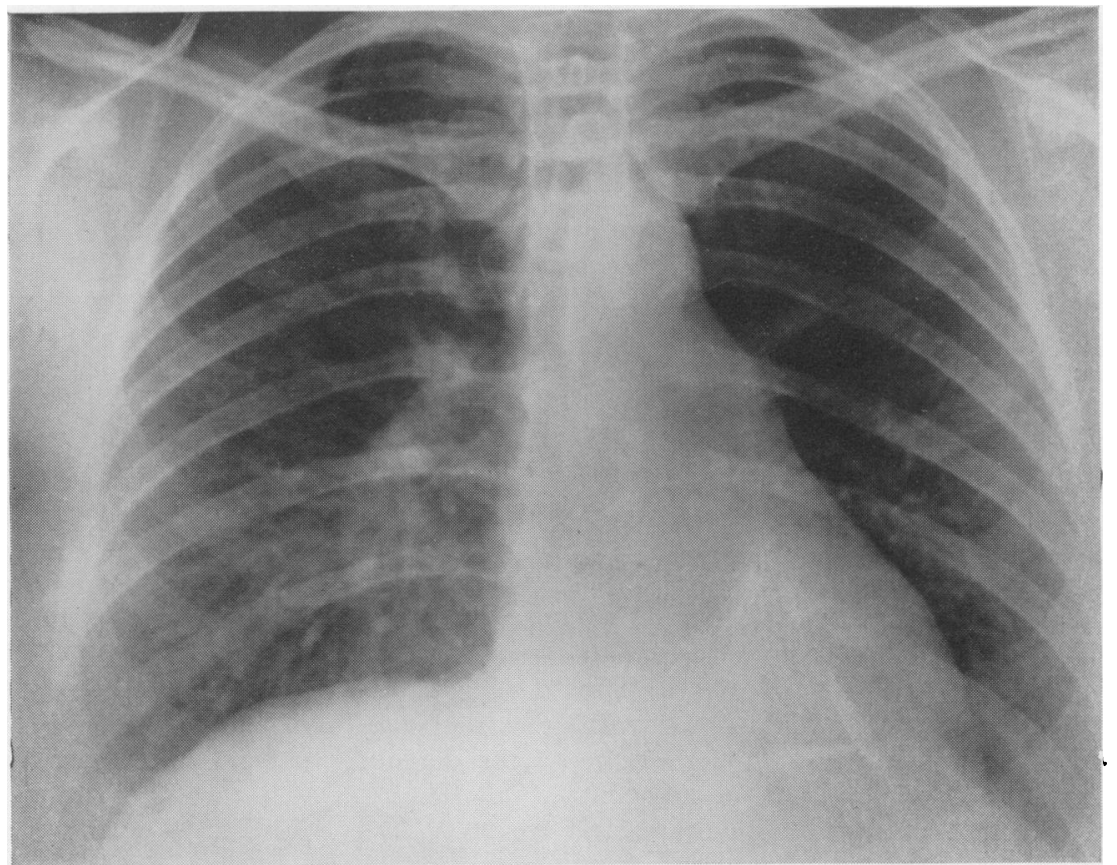

FIG. 5. (Case 4). A faint_opacity is seen through the left heart shadow.

Fig. 6. (Case 4). A.P. tomography demonstrates clearly a wedge shaped opacity in the left costovertebral angle.

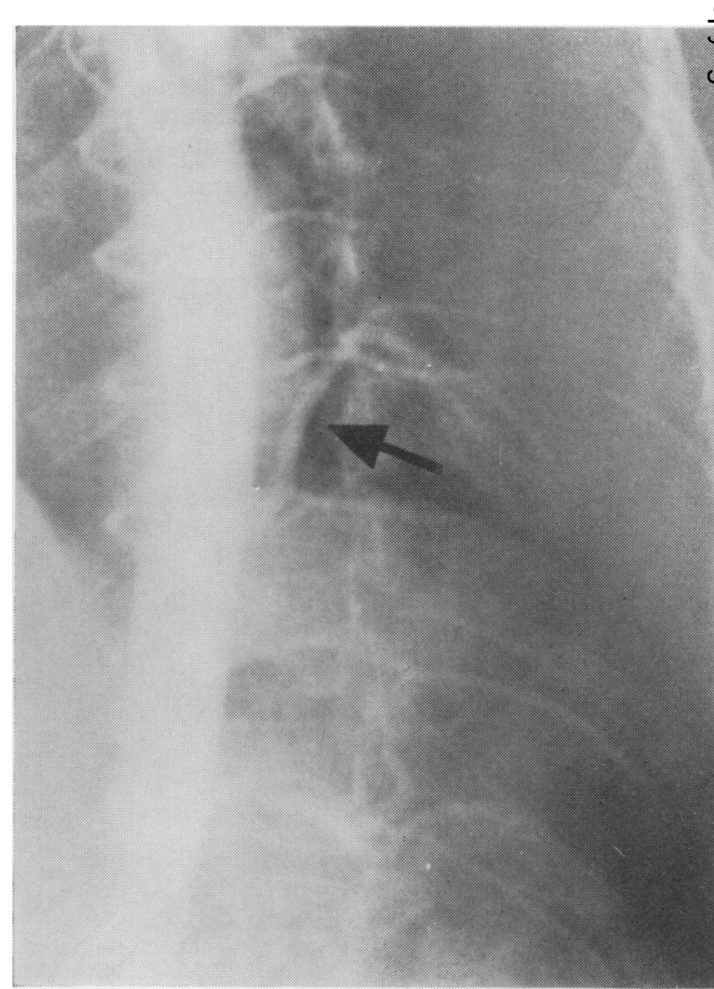

Fig. 7. (Case 4). Thoracic aortography, right posterior oblique projection. A large abnormal artery arises from the left posterior aspect of the aorta. 


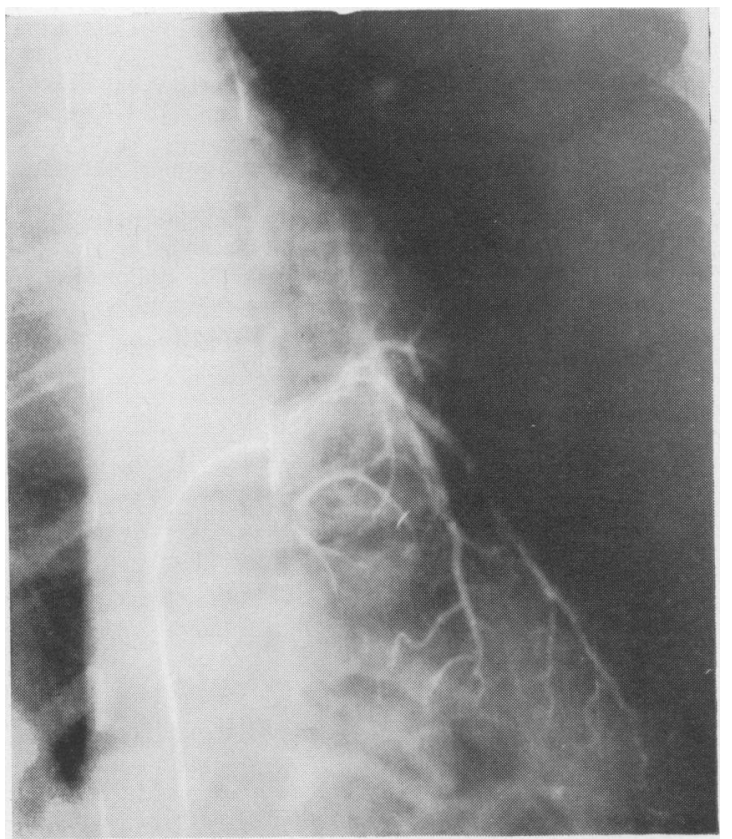

Fig. 8. (Case 4). Selective arteriogram. Late arterial phase. Note that the abnormal artery has a much wider distribution than the actual opacity seen in Figs. 5 and 6.

lous thick-walled supplying artery contained an excess of muscle.

Comment. The presentation in this case is unusual in that the presenting symptom was haemoptysis, not associated with any clinical evidence of respiratory infection. It is of interest that the abnormal artery was seen on arteriography to supply an area considerably more extensive than the opacity corresponding to the cystic mass found at thoracotomy.

\section{Discussion}

Most authorities regard intralobar pulmonary sequestration as an embryonic malformntion but there is considerable difference of opinion as to the actual pathogenesis. Pryce (1946) believes the anomalous artery to be the primary abnormality. He postulates that the normal pulmonary artery to the affected lobe is poorly developed and that the abnormal systemic artery separates the affected segment by mechanical traction. Whatever the reason for the abnormal arterial supply it seems likely that the abnormal, frequently cystic development of the segment results from its subjection to systemic arterial pressures. The excised specimen usually consists of a multilocular cystic mass the cavities of which are filled with mucus or pus. Sometimes there may be a solitary large cyst and occasionally the lesion consists of recognizable bronchial elements. Alveoli are not recognizable and there is usually no communication with the bronchial tree of the normal lung. The anomalous vessel arises from the lower thoracic aorta usually, occasionally from the abdominal aorta, or rarely from other situations (Turk \& Lindskog, 1961). This vessel has been said to have elastic rather than muscular walls (Smith, 1955). This was certainly not so in two of the cases described here. Venous drainage is generally to the pulmonary vein but may rarely be to the vena cava, to the azygos system, the portal vein or the suprarenal vein (Neilsen, 1964).

Although all four cases in this series were women it would appear that the sex incidence is about equal.

The diagnosis should be considered whenever a persisting basal opacity is encountered on a plain chest radiograph together with bronchographic appearances as described above. There may be a history of recurrent respiratory infection or of haemoptysis. A definite pre-operative diagnosis can be made only at arteriography, which demonstrates the origin, course and number of abnormal vessels. These vessels are not always readily apparent at operation and failure to identify them has on occasion had serious consequences. Arteriography, the risk of which in experienced hands is small, therefore seems justifiable as a diagnostic procedure. Since the abnormal vessel is usually fairly large, selective catheterization is feasible, allows better definition of the abnormal tissue and may demonstrate the venous drainage (Ranniger \& Valvassori, 1964 ; Boijsen \& Zsigmund, 1965).

\section{Summary}

The radiological features in four cases of intralobar pulmonary sequestration have been described. The patient commonly presents with recurrent pulmonary infection or haemoptysis, but the lesion may be discovered on routine chest radiography. The abnormal segment always occurs at the lung base posteriorly, more commonly on the left side. Radiographically it may assume any shape or size and may be cystic. By definition it does not communicate with the normal bronchial tree, but a communication may appear when infection occurs. Arteriography enables a definitive diagnosis to be made pre-operatively. Selective arteriography is advised for better definition of the arterial supply and venous drainage.

\section{References}

BoIJSEN, E. \& ZsIGMUND, M. (1965) Selective angiography of bronchial and intercostal arteries. Acta radiol. (Stockh.), 3, 513.

Douglass, R. (1948) Anomalous pulmonary vessels. J. thorac. Surg. 17, 712. 
Harris, H.A. \& Lewis, I. (1940) Anomalies of the lungs with special reference to the danger of abnormal vessels in lobectomy. J. thorac. Surg. 9, 666.

LE Roux, B.T. (1962) Intralobar pulmonary sequestration. Thorax, 17, 77.

Neilsen, P.B. (1964) Intralobar bronchopulmonary sequestration. Review of the literature and report of two cases. Amer. J. Roentgenol. 92, 547.

PRYCE, D.M. (1946) Lower accessory pulmonary artery with intralobar sequestration of lung: a report of seven cases. J. Path. Bact. 58, 457.

Pryce, D.M., Sellors, T.H. \& Blair, L.G. (1947) Intralobar sequestration of lung associated with an abnormal pulmonary artery. Brit. J. Surg. 35, 18.

RanNiger, K. \& VAlVASSORI, G.E. (1964) Angiographic diagnosis of intralobar pulmonary sequestration. Amer. $J$. Roentgenol. 92, 540.

Smith, R.A. (1955) Intralobar sequestration of the lung. Thorax, 10, 142.

Sutton, D. \& SAmuel, R.H. (1963) Thoracic aortography in intralobar lung sequestration. Clin. Radiol. 14, 317.

TURK, L.N. \& Lindskog, G.E. (1961) The importance of angiographic diagnosis in intralobar pulmonary sequestration. J. thorac. cardiovasc. Surg. 41, 299. 